\title{
Editorial:
}

\section{A Good Yield and a High Standard}

\section{Shu-Kun Lin}

Molecular Diversity Preservation International (MDPI), Saengergasse 25, CH-4054 Basel (New Address: MDPI, Matthaeusstrasse 11, CH-4057 Basel), Switzerland;

Fax:+41-61-3028918; (100700.1527@compuserve.com, New E-mail: lin@mdpi.org)

Received: 5 January 1996 / Accepted: 25 January 1996 / Published: 21 March 1996

The new journal Molecules publishes peer-reviewed research papers on synthetic chemistry and natural product chemistry. The rapid publication schedule requires that authors make sure their submitted or revised manuscripts are in the final form. In order to comply with this requirement, I considered treating the Corrigendum column as an advertisement and charging all authors who contribute to it. If Molecules were to do this, however, authors would perhaps be deterred from correcting any genuine errors found after publication of their work.

Similarly, a good-yield standard of a chemical process may also have an adverse effect. Supposing a chemist found a new reaction which worked with the syntheses of 20 compounds. It could happen that only 5 of these had satisfactory yields (higher than 75\%). Eager to publish his results, the author may include only the five good-yield examples in his paper and the results regarding the remaining 15 compounds might never be released. The editor of Molecules realizes that a good-yield standard may result in the publication of incomplete and distorted chemical information and a pitiful loss of molecular diversity.

Of course we chemists all like good yields and this aim can still remain as a high standard of synthesis of organic compounds or isolation of natural products. However, after working for 3 years in a chemical and pharmaceutical company, I now see that a high-yield is not always important, particularly at the research stage, where the acquisition of a certain amount of a target compound is the major goal. If necessary, a low-yield process can almost always be improved during further research and development. Practically only at the mass production stage is a high yield important. A pertinent example is the recent discovery of the diphenyl derivative of 1,4-diketo-pyrrolo(3,4-c)-pyrrole (DPP) [1].

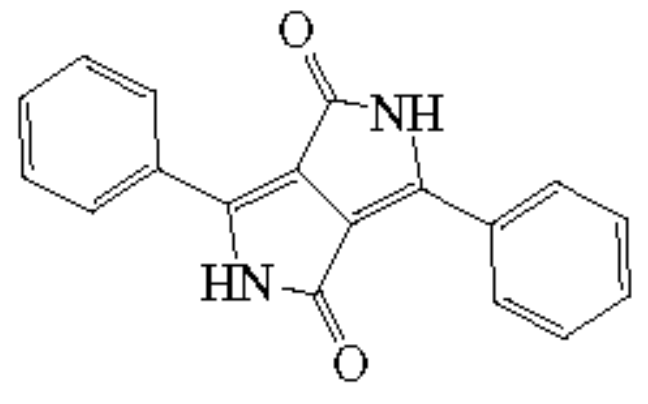

\section{DPP}

DPP was first isolated as one of several unexpected by-products and the yield was rather low [2]. The initial poor yield was significantly improved and several new methods were developed once DPP proved to be an excellent pigment [ 1]. The DPP derivatives have now become famous red pigment products (Irgazine DPP). 
I hope, therefore, that Molecules will encourage contributors to describe their research results in full, not only the high-yield examples but also all the low-yield examples, not only the structures of the target product but also the structures of isolated and characterized by-products and intermediates. The comprehensive chemical data obtained from a research topic will be presented in Molecules, and the chemical samples of the related molecules will be submitted, where possible, to an MDPI center and so be made available to the public. This procedure will make a chemist's intellectual contribution even more worthwhile, not only to further academic research, but also to support industry in the development of pharmaceutical, agrochemical and other chemical products. This is the high standard that Molecules strives for.

\section{References and Notes}

1. Zollinger, H. Color Chemistry, VCH: Weinheim, 1991; 241-244.

2. Farnum, D. G.; Mehta, G.; Moore, G. G. I.; Siegal, F. P. Tetrahedron Lett. 1974, 2549. 